\title{
Adding cognitive therapy to minimal psychiatric care prevents short term, but not long term, relapse in people with bipolar disorder
}

Lam DH, Hayward P, Watkins ER, et al. Relapse prevention in patients with bipolar disorder: cognitive therapy outcome after 2 years. Am J Psychiatry 2005;162:324-9.

$Q$ Does cognitive therapy plus minimal psychiatric care reduce the risk of long term relapse in people with bipolar disorder?

METHODS

-

Design: Long term follow up of a randomised controlled trial.

Allocation: Concealed.

Blinding: Single blinded.

Follow up period: 2.5 years (1 year results reported previously).

Setting: Unclear.

Patients: 103 people (mean age 44 years) with bipolar disorder (DSM-IV diagnosis). Inclusions: two or more bipolar episodes in the previous two years or three episodes in the last five years. Exclusions: current substance abuse or suicidal behavior.

$\mathbf{R}_{\mathbf{C}}$

Intervention: Cognitive therapy (12-18 sessions within the first six months and two booster sessions) plus minimal psychiatric care (mood stabilisers with regular outpatient psychiatric follow up) or minimal psychiatric care alone.

国坚

Outcomes: Relapse rates; time in relapse.

吅 $\square$

Patient follow up: Not reported.

\section{MAIN RESULTS}

Time in relapse: Cognitive therapy (CT) plus minimal psychiatric care significantly reduced time spent in relapse compared with minimal psychiatric care alone over 2.5 years, including the last 1.5 years of follow up (time in relapse over 2.5 years: 95.3 days with CT plus minimal psychiatric care $v 201$ days with minimal psychiatric care alone, $\mathrm{p}=0.005$; time in relapse over the last 1.5 years: 64.1 days with CT plus minimal psychiatric care $v 107.5$ days with minimal psychiatric care alone, $\mathrm{p}=0.04)$. Relapse rates: Over 2.5 years, cognitive therapy plus minimal psychiatric care significantly reduced relapse rates for bipolar and depressive episodes, but not manic/hypomanic episodes compared with minimal psychiatric care alone (bipolar episodes: HR 0.50, 95\% CI 0.29 to 0.85 ; depressive episodes: HR $0.38,95 \%$ CI 0.19 to 0.75 ; manic/hypomanic episodes: HR $0.71,95 \%$ CI 0.38 to 1.35 ). However, there was no significant difference in relapse rates over the last 1.5 years of follow up (proportion of people with one or more relapse: $26 / 45$ (57.8\%) with CT plus minimal psychiatric care $v 32 / 46(69.6 \%)$ with minimal psychiatric care, $\mathrm{p}$ value not reported).

For correspondence: Dr Lam, Pyschology Department (P077), Henry Wellcome Building, Institute of Pyschiatry, De Crespigny Park, London SE5 8AF, UK; spjtdhk@iop.kcl.ac.uk

Sources of funding: none declared.

\section{CONCLUSIONS}

Cognitive therapy plus minimal psychiatric care reduces time in relapse over 2.5 years compared with minimal psychiatric care alone. Cognitive therapy reduces overall relapse rates in the first year, but not after the first year.

\section{Commentary}

Tre he prognosis for bipolar disorder remains poor with frequent relapses and recurrences along with significant interepisode symptomatology. ${ }^{1}$ Despite maintenance pharmacotherapy, the chance of recurrence within the first year is more than $50 \%$, increasing to $90 \%$ after five years. ${ }^{2} 3$ The limited success of medications has provided the impetus to augment maintenance pharmacotherapy with psychosocial interventions in an effort to improve treatment outcome. These efforts parallel similar earlier initiatives for schizophrenia. The report by Lam et al adds to a few studies evaluating other psychosocial interventions ${ }^{4-6}$ supporting psychotherapy efficacy in the management of bipolar disorder. However, sample sizes are often small, random assignment is rare, and outcome raters are often not blind to treatment condition.

Lam et al randomly assigned 103 people with bipolar I disorder, vulnerable to relapse, to either cognitive therapy (CT) plus medication or medication alone for a period of six months. Participants were assessed for two years by raters blinded to treatment assignment. CT significantly reduced relapse rates during the first 12 months, however, there was no reduction in relapse over the last 18 months of the study period. Nevertheless, over the last 18 months of the study the CT group spent $12 \%$ fewer days in bipolar episodes and demonstrated improved mood ratings, social functioning, coping with bipolar prodromes, and reduced dysfunctional cognitions.

Whether the current intervention is considered successful depends in part on how outcome is defined. These results provide preliminary support for adding CT to current treatment regimens, as CT provides some benefit with little risk. Future research replicating or improving upon the results of this study (for example, by adding maintenance CT to sustain treatment benefits long term) and demonstrating the specificity of CT's efficacy compared to other psychosocial interventions would provide additional incentive for combining $\mathrm{CT}$ with pharmacotherapy. Mark A Lau, PhD, CPsych

Centre for Addiction and Mental Health, Department of Psychiatry, University of Toronto, Toronto, Canada

1 Solomon DA, Keitner GI, Miller IW, et al. Course of illness and maintenance treatments for patients with bipolar disorder. J Clin Psychiatry 1995;56:5-13.

2 Keller MB, Lavori PW, Coryell W, et al. Bipolar I: a five-year prospective follow-up. J Nerv Ment Dis 1993;181:238-45.

3 Tohen $M$, Waternaux CM, Tsuang MT. Outcome in mania: a 4-year prospective follow-up of 75 patients utilizing survival analysis. Arch Gen Psychiatry 1990;47:1106-11.

4 Colom F, Vieta E, Martinez-Aran A, et al. A randomized trial on the efficacy of group psychoeducation in the prophylaxis of recurrences in bipolar patients whose disease is in remission. Arch Gen Psychiatry 2003;60:402-7.

5 Miklowitz DJ, Simoneau TL, George EL, et al. Family focused treatment of bipolar disorder: 1-year effects of a psychoeducational program in conjunction with pharmacotherapy. Biol Psychiatry 2000;48:582-92.

6 Frank E. Interpersonal and social rhythm therapy prevents depressive symptomatology in bipolar I patient. Bipolar Disorder 1999;1(Suppl):13. 